\title{
The Evolution of Key Technologies of Mobile Communication Systems for High-speed Railway \\ Feng Pan
}

\author{
Sichuan Vocational College of Information Technology, Sichuan, GuangYuan, 628040, \\ China
}

Key words: high-speed railway; mobile communication; GSM-R; evolution

Abstract:With the rapid economic development, science and technology are gradually progressing, high-speed railway attracts more and more attention. Since the birth of the high-speed railway , along with the demand for mobile communications, part of the demand from the high-speed train train control and scheduling system, which is the core of high-speed train; the other part is from the demand of passengers. In the past, GSM-R technology is more mainstream high-speed railway mobile communication technology, in addition, TGV and ICE applications such as mobile communication system is also a typical communication technology. In the future, with the $5 \mathrm{G}$ era approaching, the high-speed railway communication technology based on $5 \mathrm{G}$ will become the trend, it may soon replace GSM-R technology to become a new generation of mobile communication key technology, and high-speed railway wireless network access system will also be put into operation in the near future. Looking forward to through research and discussion, high-speed railway communication technology can be further developed and extended.

\section{Introduction}

The world's most advanced high-speed wheel-rail transportation system, many of which are or will be developing high-speed train wireless communication system, in the provision of train control train dispatch business carrying, but also to provide passengers with the necessary communications and Internet access services. In addition to GSM-R (Global System for Mobile communications for Railway), the more representative of the high-speed rail mobile communications systems are Germany ICE (Inter City Express) and Telefunken, France TGV (Train a Grande Vitesse) and Thalys and the Japanese Shinkansen (Shinkansen) on the use of the corresponding technology. Andrew offers a unique wireless access solution for the German ICE Intercity Express, the core of which is the car repeater. The car repeater can amplify the signal received from the nearby network, reduce the signal due to the attenuation of the train metal shell, improve the quality of wireless access. German Telefunken wireless communication systems use proprietary protocol technology, and there is not much detail about the technology. The French TGV satellite access scheme uses a wireless access technology based on two-way satellite systems: vehicle access is shared by satellites and terrestrial WiFi repeaters covering tunnels and stations. When satellites can not cover trains, WiFi networks will take over wireless access so that upload and download data are not interrupted.

Japan Shinkansen early WiFi leak cable program and the recent WiMax (Worldwide interoperability for Microwave access) leak cable program in the wireless access widely used coaxial leak cable technology, the coaxial leak cable installed in the track along the WiFi system for the passengers information system (Passenger Information System, PIS) to provide transmission channels, WiMax system for the passenger internet access to provide transmission channels. The mainstream GSM-R was launched by the European countries and was originally deployed in Germany, Italy, the Netherlands, Norway, Sweden and other countries. It is mainly deployed in European countries as well as China and India in Asia.Table.1 is a detailed comparison of high-speed rail mobile communication systems in countries or regions ${ }^{[1-2]}$. 
Tab.1Performance Comparison of Main Railway Information System

\begin{tabular}{ccccc}
\hline & \multicolumn{4}{c}{ Country or region } \\
\cline { 2 - 5 } Express train & Germany & France & Japan & Taiwan, China \\
\cline { 2 - 5 } & ICE & TGV/Thalys & ShinkansenN700 & Taipei \\
\hline $\begin{array}{c}\text { Maximum } \\
\text { speed(km/H) }\end{array}$ & 280 & 320 & 300 & 300 \\
\hline $\begin{array}{c}\text { Wireless access } \\
\text { system }\end{array}$ & GSM-R & GPRS/UMTS/WiFi & $\begin{array}{c}\text { Coaxial leak } \\
\text { cable/WiFi }\end{array}$ & WiMax \\
\hline $\begin{array}{c}\text { Average transfer } \\
\text { rate(Mbps) }\end{array}$ & $0.05 / 0.05$ & $2 / 0.512$ & $2 / 1$ & $10 / 30$ \\
\hline
\end{tabular}

\section{GSM-R Technology}

\subsection{The maturity and historical mission of GSM-R technology}

GSM-R has its undeniable, epoch-making significance and role, through the mature, long-term commercial and standardized GSM technology into the railway system, improve the efficiency of railway operation and management, and save the costs of construction and operation Its support for roaming (such as switching) can automatically control the train during the entire run, making it to be possible for the rail system to use unified voice and data transmission platforms ${ }^{[3-4]}$.

But GSM-R is the technology of the eighties and nineties of last century, today, wireless communications have made revolutionary progress and breakthrough, various shortcomings and bottlenecks are inevitably exposed, the narrow band characteristics only meet the existing low data rate of the train control train scheduling business needs, the allocation of $4 \mathrm{MHz}$ bandwidth to make this situation to be worse. And GSM-R can not carry the high-speed railway future intelligent scheduling, video surveillance and operation and management of high data rate business and passenger broadband access services needs. Therefore, the international union of railways (International Union of Railways) considers and deploys GSM-R the next 5 to 10 years of evolution strategy according to the GSM-R technology life cycle. GSM-R manufacturers may also be in 2025 or so to stop the GSM-R equipment upgrades and maintenance, when the equipment supply chain will be interrupted. In addition, according to the 15 years of communication equipment overhaul cycle, the existing GSM-R equipment used in 2024 will face evolution or replacement issues.

\subsection{Key Technologies of GSM-R Technology Recent Evolution}

The train control and dispatching business is the main business scope of the high-speed railway mobile communication system, but the high data rate service such as intelligent dispatching and video monitoring in the future will become the main service content and will also expand the service for the passengers. Information security is one of the key technologies in the future of dedicated mobile communication technology. If security can not be dealt with completely, high-speed railways will be difficult to achieve a passenger-oriented service base and will not be able to achieve a greater profitability. It is generally believed that the recent evolution of GSM-R technology is divided into two phases. The initial GSM-R and LTE-R system co-exist, the former is responsible for train control and other safety-related business transmission, which is responsible for video surveillance and other non-security services and passenger broadband access services and other data transmission. Later, more services will be carried by the LTE-R network, and eventually all will be switched to the LTE-R network $^{[5]}$.

\section{The future evolution route of mobile communication technology for high-speed railway}

\subsection{G high-speed railway mobile communication technology}

All the monitoring data and passenger information service requests are forwarded to the train control system and the internet server through the relay nodes. For the downlink, the base station transmits the train control service (e.g., mobile authorization information) and the passenger 
information data to the relay node. The train control information is forwarded to the vehicle control equipment via the wired link. The passenger information service is forwarded to the passengers through the second jump wireless link, and the link layer adopts the technology to improve the reliability of the transmission. As the train control system is a safe system, its safety integrity level needs to reach the level, such as the probability of failure needs to be achieved, so the train control business error rate requirements higher than the passenger package requirements. As shown in Fig.1

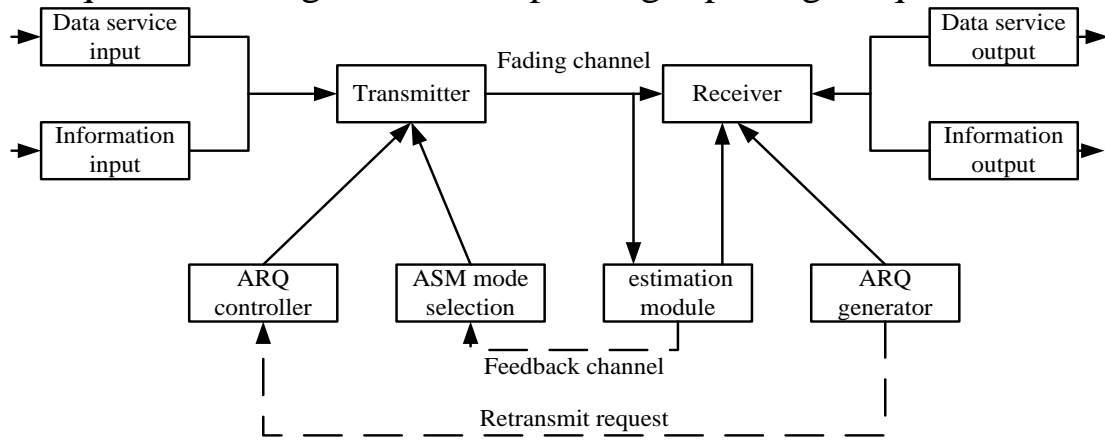

Fig.1 Link transmission scheme

There are three main ways to improve the system capacity: to expand the system bandwidth, increase the spectral efficiency and increase the network density, the most direct and effective way is to increase the system bandwidth. But wireless spectrum, as an extremely limited and valuable resource, requires the authorization of the relevant state departments, so it is difficult to obtain more licenses, or to pay a huge economic cost. Therefore, the rational and effective use of non-license band is $5 \mathrm{G}$ rail mobile communication system to increase the system bandwidth and improve the system capacity of the important method. However, the non-license band is generally in the spectrum and the license band is far away, the transmission characteristics of a large difference, so the need for non-license band design and license band different wireless transmission technology, that is spectrum fusion technology. In order to make the spectrum fusion technology in the high-speed rail scene to be achievable, it is necessary to solve the three major problems of non-license band instability, non-license and license band transmission characteristics difference and interference coordination ${ }^{[6]}$. The main principle of spectrum fusion is shown in Fig.2.

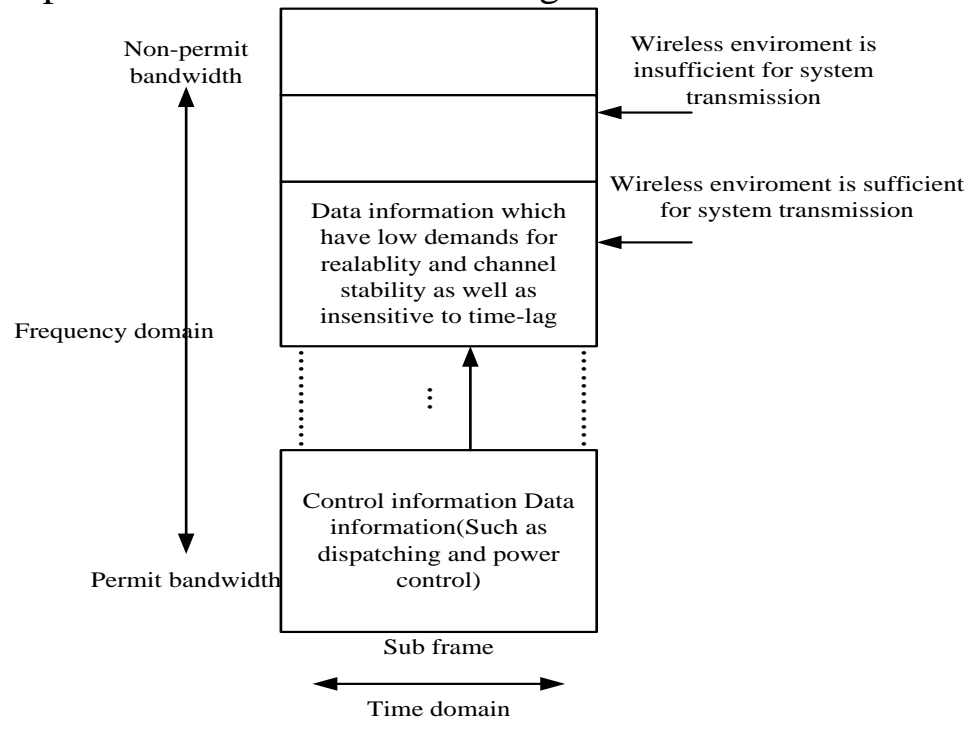

Fig.2 Schematic diagram of frequency spectrum integration of heterogeneous networks

\subsection{High-speed railway wireless network access system}

The rapid development of mobile internet technology, but it is also possible for passengers in the station and car to enjoy internet under the broadband access service. Car passengers directly with the roadside cellular base station to establish a connection is the most simple way to access, the base station and user terminal hardware and software upgrades require less configuration. However, the 
general passenger terminal equipment processing capacity and power is limited, due to the larger body penetration loss, group switching signaling storm and other issues, resulting in a larger terminal call drop. With the evolution of the public network and wireless LAN technology, the train passenger wireless communication system should also consider the forward-looking and advanced, need to re-establish high-speed railway scene to get off the broadband data access wireless data transmission link. The introduction of an in-vehicle access system (Femto system), WiFi and other in-car access system in the train provides a good solution for these problems. Based on the above functional requirements, there are still a series of technical standards and key technologies to be solved, including the next generation of mobile communication networks and wireless local area network access, vehicle wireless connection, large-capacity concurrent signaling storm, vehicle connection equipment and cabins Interference between devices into the coordination and so on ${ }^{[7]}$.

\subsection{Power carrier communication Technology}

In recent years, some scholars have proposed the use of high-speed railway power line carrying high-speed railway mobile communication technology. The use of power transmission lines for power carrier communication technology has been well applied at home and abroad, with the help of this concept and leakage cable transmission technology, some scholars have also proposed the use of high-speed railway power line as a new wireless communication transmission The principle is the use of microwave "skin effect", with the catenary as an antenna, just like the leakage cable, the base station signal across the entire railway line. The broadband signal generated by the base station is fed into the power cable through the coupler, and the transmission power is dynamically configured as necessary so that the interference generated by the distance is far from being absorbed by the absorber. The program can be installed in the train roof antenna, while maintaining the receiving antenna and the power cable a certain non-contact wireless transmission distance. The technology has a clear cost advantage, but the key technology has not yet been fully verified.

\section{Summary}

The rapid development of high-speed railway, the wireless communication system between the car put forward higher requirements, the current narrow band GSM-R system will gradually fade out of the stage of history, high-speed railway mobile communication system is about to LTE-R. In the design of LTE-R, on the one hand, in order to improve the safety of the train operation, need to consider the installation of video surveillance equipment, real-time monitoring of the train. On the other hand, also need to consider the passenger mobile internet business needs. And these business needs in the long run, will soon be a challenge to LTE-R system. Fortunately, we can learn from the public network 5G system to extend the bandwidth of high-frequency band ideas, according to the layout of high-speed rail mobile communication system long-term evolution line. In the future development of high-speed railway mobile communication system, the key technology process, but also need to enhance the service function to protect the needs of passengers using wireless networks, science and technology to support the development of the scale of the industry to promote people's quality of life requirements.

\section{References}

[1] Tingting G, Bin S. A high-speed railway mobile communication system based on LTE[C]//Electronics and Information Engineering (ICEIE), 2010 International Conference On. IEEE, 2010, 1: V1-414-V1-417.

[2] Liu L, Tao C, Qiu J, et al. Position-based modeling for wireless channel on high-speed railway under a viaduct at $2.35 \mathrm{GHz}[\mathrm{J}]$. IEEE Journal on Selected Areas in Communications, 2012, 30(4): 834-845.

[3] Wang J, Zhu H, Gomes N J. Distributed antenna systems for mobile communications in high speed trains[J]. IEEE Journal on Selected Areas in Communications, 2012, 30(4): 675-683..

[4] He R, Zhong Z, Ai B, et al. An empirical path loss model and fading analysis for high-speed railway viaduct scenarios[J]. IEEE antennas and wireless propagation letters, 2011, 10: 
808-812.

[5] Karimi O B, Liu J, Wang C. Seamless wireless connectivity for multimedia services in high speed trains[J]. IEEE Journal on selected areas in communications, 2012, 30(4): 729-739..

[6] Wang C X, Haider F, Gao X, et al. Cellular architecture and key technologies for 5G wireless communication networks[J]. IEEE Communications Magazine, 2014, 52(2): 122-130..

[7] Ai B, Cheng X, Kürner T, et al. Challenges toward wireless communications for high-speed railway[J]. IEEE Transactions on Intelligent Transportation Systems, 2014, 15(5): 2143-2158.. 